\title{
In vitro evaluation of the structural and bioaccessibility of kenaf seed oil nanoemulsions stabilised by binary emulsifiers and $\beta$-cyclodextrin complexes
}

\begin{abstract}
Kenaf (Hibiscus cannabinus L.) seed oil contains high nutrition value, suggesting its potential applications in food and nutraceutical fields. However, the poor water solubility and stability strictly limit its applications. A good physical stability of kenaf seed oil-in-water nanoemulsions stabilised by sodium caseinate, Tween 20 and $\beta$-cyclodextrin complexes were produced using high pressure homogeniser. A simple model of two-stage dynamic in vitro digestion was employed to investigate the releasing rate of bioactive compounds from kenaf seed oil-in-water nanoemulsions, compared to unencapsulated bulk oil. The particles size and structural changes during digestion were also evaluated. Digested nanoemulsions showed good lipid digestion (85.25\%), good bioaccessibility of antioxidants (tocopherols and total phenolic contents) and lower degradation rate of phytosterols compared to digested bulk oil. This study provides good information about the characteristic and release behaviour of formulated kenaf seed oil-in-water nanoemulsions, which is important for the future application in food and nutraceutical industries.
\end{abstract}

Keyword: Kenaf (Hibiscus cannabinus L.) seed oil; Lipolysis; Releasing behaviour; Confocal microscopy; Tocopherols; Phytosterols 